\title{
EDITORIAL
}

\section{Prescribing and guidelines: both must improve to combat antimicrobial resistance}

\author{
M. Woodhead
}

$\mathbf{T}$ he problem is the development and spread of antimicrobial resistance in common bacterial pathogens. The cause is excessive exposure of these bacteria to antimicrobial agents. The solution is to reduce antimicrobial exposure, the most important element of which is to reduce human use.

Most human exposure occurs through antimicrobial prescription in the community, the most common reason for which is presumed respiratory infection. Human exposure varies widely between European countries, with the same patient being, on average, four times more likely to receive an antibiotic in France compared to the Netherlands [1]; a difference that cannot be explained by the scientific evidence base. Clearly there are respiratory infections where antimicrobial use is an essential part of management, so the name of the game is to promote "prudent" or "appropriate" antimicrobial prescribing. There is no single strategy that will lead to prudent prescribing, as elements such as agreement as to what is or is not prudent, medical education and public education all play a part. One important element is medical education through the use of clinical practice guidelines. The European Council has recommended that member states, as part of control and preventive measures (for antimicrobial resistance), "develop[ing] evidence-based principles and guidelines on good practice for the management of communicable diseases, to maintain the effectiveness of antimicrobial agents" [2]. European Respiratory Journal (ERJ) readers will be aware of the guidelines for the management of lower respiratory tract infections developed jointly by the European Respiratory Society (ERS) and the European Society of Clinical Microbiology and Infectious Diseases (ESCMID), part of which was directed to the promotion of appropriate antimicrobial practice for adult lower respiratory tract infection (LRTI) in the community [3]. The guidelines recommended that antibiotics should be considered in LRTI in the community only in the following settings: suspected or definite pneumonia, selected exacerbations of COPD and in those aged $>75$ yrs with one or more of fever, cardiac failure, insulin-dependent diabetes mellitus or serious neurological disorder. Of course for any guideline to impact on antimicrobial prescribing and resistance, clinical practice must follow, or at least be congruent with, the guideline.

CORRESPONDENCE: M. Woodhead, Manchester Royal Infirmary and University of Manchester Oxford Road, Manchester, UK. E-mail: mark.woodhead@cmft.nhs.uk
In this issue of the ERJ, WoODS et al. [4] have, for the first time, attempted to describe the congruence of antibiotic prescribing for LRTI in the community in 13 European countries with the ERS/ESCMID LRTI guidelines. These authors studied 2,690 adult LRTIs using 14 general practice networks involved in the EU-funded GRACE network (Genomics to Combat Resistance against Antibiotics in Community-acquired LRTI in Europe; www.grace.eu). Overall congruence with recommendations was good: in 528 patients, the guideline recommended that an antibiotic was not indicated and it was not prescribed, and in 1,217 an antibiotic was indicated and was prescribed; a total congruence of $64.9 \%$. In this era of antibiotic overuse, perhaps the most surprising finding in this study was that there were twice as many situations where an antibiotic was indicated than where one was not, and that the biggest area of noncongruence with the guideline recommendations was in the $698(25.9 \%)$ where the guidelines would recommend an antibiotic, but one was not prescribed. Linked to this the authors estimated that an antibiotic could have been justified according to the guidelines in $71 \%$ of patients while only $54.4 \%$ actually received an antibiotic. In other words, if the guidelines had been followed exactly, an increase in antibiotic prescribing would have been found!

This is, at first sight, counterintuitive, so let us explore the possible reasons for this.

The first possible explanation is that there are flaws in the study. Could the study patient population have been biased towards a group of LRTIs more likely to merit antimicrobial therapy? In favour of this is the observation by WooDs et al. [4] that by using the guideline criteria, $70.8 \%$ of patients could have been considered to have suspected or definite pneumonia; a much higher proportion than the $18 \%$ described in an earlier study of prescribing for adult LRTI in the community in Europe [5]. The authors also note that the $\sim 20 \%$ of recruited patients that were excluded from the analysis were younger and were less frequently prescribed antibiotics.

An alternative explanation is that the guideline recommendations are wrong, or more accurately at the very least, insufficiently precise. Guideline recommendations are usually only as robust as the evidence on which they are based and a quick look at the ERS/ESCMID guideline shows that the antibiotic recommendation for LRTI in the community was developed by consensus rather than having a strong evidence base. Strong evidence in the form of systematic reviews and randomised clinical trials are few in the setting of LRTI in the community. The only systematic reviews cover the topics of 
acute bronchitis [6] and exacerbations of COPD [7]. While the latter review concluded that antibiotics are of benefit in more severely ill patients, its conclusions are limited by the small number of studies included, often by their small size and, in particular, by their heterogeneity. Both systematic reviews are limited by the fundamental inaccuracy of clinical symptoms and signs which the doctor in the community must rely on, related to variability in both their identification and interpretation, to separate pathologically different medical conditions. Making a robust diagnosis of pneumonia in the community based on clinical symptoms and signs is almost impossible [8] and this may explain why such a large proportion of patients could have been considered to have pneumonia in the study by WooDs et al. [4]. It is unlikely that we will be able to make these diagnoses more accurate by further evaluation of clinical features, although assessment of prognostic factors $[9,10]$ may help to identify the groups in which randomised antibiotic trials are likely to be most beneficial. A further "nail in the coffin" of clinical featurebased antibiotic prescription in LRTI is provided by a separate analysis of the same dataset by BUTLER et al. [11] in this issue. Sputum purulence has long been accepted as a correlate of bacterial infection and a need for antibiotic prescription. However these authors, while finding that those with purulent sputum were three times more likely to receive antibiotics, also found that recovery was identical regardless of sputum colour or whether antibiotics were prescribed or not. Even a measure of unwellness did not correlate with antibiotic benefit. As the authors acknowledge, this was not a double-blind randomised trial and therefore lacked methodological rigour for the determination of a true intervention effect or lack of it. It was, however, potentially closer to real clinical practice and included a very large patient population. Complete abandonment of antibiotics in uncomplicated LRTI outside hospital would be best for antibiotic resistance and might be best for most patients, but probably not all. However, further studies are required before we can consider this. In addition to the need for more data, the general practitioner also has the disadvantage of a lack of easily available and widely accepted alternative treatments for this common clinical scenario. Delayed prescribing and information leaflets have shown promise as alternatives to antibiotics [12], but currently have the major disadvantage of lack of widespread acceptance in the general population. Of course a subgroup of patients with LRTI may still gain benefit from antibiotics, but how is the busy general practitioner to identify them, if sputum purulence is confirmed to be of no value? At present the groups defined in the guideline, as listed above, are probably those most likely to benefit from antibiotics, but we lack hard evidence to strongly support these recommendations at present. One area of promise is the potential added value of near-patient inflammatory marker monitoring. This takes us away from the "what is the diagnosis?" approach and moves towards the "who is likely to benefit from an antibiotic?" approach, which is the clinical question actually under scrutiny in most cases. The promise has yet to be realised, however, as two metaanalyses have given conflicting assessments on the value suggested by studies so far [13, 14].

Of course, the guidelines used as reference in this study are European and not national. It may be that national or local guidelines make different recommendations from the European document. It was beyond the scope of the study to assess whether any guidelines were actually referred to by the prescribing doctors in this study and the authors were careful to use the term congruence so as not to imply that guidelines were or were not being followed. Another issue is the terminology of the guidelines. A recommendation to "consider" the use of antibiotics is not the same as a recommendation that antibiotics "must" be used. The absence of such unambiguous recommendations again reflects the lack of good research evidence for both harm and benefit that would be required for a "must do" or "must not do" recommendation. "Consider" does not mean "do it in everyone", but how considerations are made will vary between individual doctors for a variety of reasons, including training, individual experience and the exact nature of the situation of the patient in front of the doctor.

For now we are left with heterogeneity of antibiotic prescribing across Europe. WooDs et al. [4] found this in their study, with $88 \%$ of patients receiving antibiotics in Bratislava compared to $18 \%$ in Barcelona. There would be a concern if outcomes were worse in those countries with less antibiotic prescription, but this does not appear to be the case. The GRACE network's own data showed no clinically important outcome differences associated with such variations in prescribing practice [15]. While there are studies which have purported to show the converse, i.e. increased mortality associated with reduced antimicrobial prescribing, the methodological robustness of such studies, usually involving unlinked databases, is open to question [16].

The congruence with guideline recommendations found by Woods et al. [4] is encouraging, but the European heterogeneity remains a concern. Antibiotic prescribing can become more prudent. This applies to all countries, but those with the highest prescribing burden, although having more work to do, can be reassured that this can be done without harm to patients. The guidelines must be improved. This can only happen with more robust evidence. The results of the antibiotic study from the GRACE network is very helpful but, as so often is the case, pose as many questions as they answer. A revision of the ERS/ESCMID LRTI guidelines is in development, but it is likely to be a few more revisions yet before we have all the answers about how community LRTIs should be managed.

\section{STATEMENT OF INTEREST}

A statement of interest for M. Woodhead can be found at www.erj. ersjournals.com/site/misc/statements.xhtml

\section{REFERENCES}

1 Goossens H, Ferech M, Vander SR, et al. Outpatient antibiotic use in Europe and association with resistance: a cross-national database study. Lancet 2005; 365: 579-587.

2 Summaries of EU Legislation. Council Recommendation of 15 November 2001 on the Prudent Use of Antimicrobial Agents in Human Medicine. Brussels, EUR-Lex, 2002. Available from: http:/ / eur-lex.europa.eu/smartapi/cgi/sga_doc?smartapi!celexplus!prod! DocNumber\&lg $=$ en\&type_doc $=$ COMfinal\&an_doc $=2001 \& n u$ doc $=333$.

3 Woodhead M, Blasi F, Ewig S, et al. Guidelines for the management of adult lower respiratory tract infections. Eur Respir J 2005; 26: $1138-1180$. 
4 Wood J, Butler CC, Hood K, et al. Antibiotic prescribing for adults with acute cough/lower respiratory tract infection: congruence with guidelines. Eur Respir J 2011; 38: 112-118.

5 Huchon GJ, Gialdroni-Grassi G, Leophonte P, et al. Initial antibiotic therapy for lower respiratory tract infection in the community: a European survey. Eur Respir J 1996; 9: 1590-1595.

6 Smith SM, Fahey T, Smucny J, et al. Antibiotics for acute bronchitis. Cochrane Database Syst Rev 2004; 4: CD000245.

7 Ram FS, Rodriguez-Roisin R, Granados-Navarrete A, et al. Antibiotics for exacerbations of chronic obstructive pulmonary disease. Cochrane Database Syst Rev 2006; 2: CD004403.

8 Graffelman AW, le Cessie S, Knuistingh Neven A, et al. Can history and exam alone reliably predict pneumonia? J Fam Pract 2007; 56: 465-470.

9 Bont J, Hak E, Hoes AW, et al. A prediction rule for elderly primary-care patients with lower respiratory tract infections. Eur Respir J 2007; 29: 969-975.

10 Bont J, Hak E, Hoes AW, et al. Predicting death in elderly patients with community-acquired pneumonia: a prospective validation study reevaluating the CRB-65 severity assessment tool. Arch Intern Med 2008 14, 168: 1465-1468.

11 Butler CC, Kelly MJ, Hood K, et al. Antibiotic prescribing for discoloured sputum in acute cough/lower respiratory tract infection. Eur Respir J 2011; 38: 119-125.

12 Little P, Rumsby K, Kelly J, et al. Information leaflet and antibiotic prescribing strategies for acute lower respiratory tract infection: a randomized controlled trial. JAMA 2005; 293: 3029-3035.

13 van der Meer V, Neven AK, van den Broek PJ, et al. Diagnostic value of $C$ reactive protein in infections of the lower respiratory tract: systematic review. BMJ 2005; 331: 26.

14 Falk G, Fahey T. C-reactive protein and community-acquired pneumonia in ambulatory care: systematic review of diagnostic accuracy studies. Fam Pract 2009; 26: 10-21.

15 Butler CC, Hood K, Verheij T, et al. Variation in antibiotic prescribing and its impact on recovery in patients with acute cough in primary care: prospective study in 13 countries. BMJ 2009; 338: b2242.

16 Winchester CC, Macfarlane TV, Thomas M, et al. Antibiotic prescribing and outcomes of lower respiratory tract infection in UK primary care. Chest 2009; 135: 1163-1172. 